\title{
ANALYSIS OF PLATE BENDING BY THE BOUNDARY ELEMENT METHOD CONSIDERING PASTERNAK-TYPE FOUNDATION
}

\author{
V. J. Karam ${ }^{1}$, M. Altoé ${ }^{1}$, N. S. Ribeiro ${ }^{1}$ \\ ${ }^{1}$ Laboratório de Engenharia Civil, Centro de Ciência e Tecnologia, Universidade Estadual do \\ Norte Fluminense Darcy Ribeiro (UENF), Avenida Alberto Lamego, 2000, Parque Califórnia, \\ 28013-602, Campos dos Goytacazes, RJ, Brazil (corresponding author: vjkaram@uenf.br).
}

\begin{abstract}
The Boundary Element Method (BEM) is used in this work to the analysis of plates resting on Pasternak-type foundations, considering Reissner's theory. The used approach considers the fundamental solution already employed in the analysis of plates by the BEM. Integral equations that are used to solve the problem are presented, in which domain integrals related to soil reactions are included. The region situated over the foundation is discretized into constant internal cells and the integrals related to the cells are transformed into integrals over the boundary of these cells. Since the soil reactions are also unknowns to the problem and these forces are written in terms of transverse displacements and their derivatives, additional equations written for domain points situated in the cells are considered. In order to define additional equations to the original system, integral equations for transverse displacements at internal points and integral equations for the second derivatives of these transverse displacements are added to the system and these displacements and respective derivatives are also considered as unknowns to the resulting system. Continuous and discontinuous quadratic boundary elements are used in the numerical implementation. Some numerical examples are presented and results are compared with those obtained by other authors with different approaches.
\end{abstract}

Keywords: Reissner's Theory, Boundary Element Method, Pasternak-Type Foundation.

\section{INTRODUCTION}

Plate bending analysis by the Boundary Element Method (BEM) using Reissner's theory was initially developed by Van der Weeën [9]. Some authors who have studied plates on elastic supports with the BEM are: Paiva [4], Rashed et al. [5] and Ribeiro and Karam [8], using Winkler's model; and Jianguo et al. [2], Rashed et al. [6] and Altoé and Karam [1], using Pasternak's model.

Reissner's theory [7] allows the analysis of thin and thick plates and considers the three physical boundary conditions concerned with the problem, leading to results more accurate near edges and corners than those obtained from Kirchhoff's theory, which considers only two boundary conditions in each edge and, in consequence, is limited to thin plates. 
The Boundary Element Method is used, in this work, to the numerical solution, considering Reissner's theory and also the soil reaction with a Pasternak-type model.

The approach carried out in this work uses a fundamental solution already employed in plate bending analysis by the BEM [3,9] and additional equations to the original system, written for points of the contact region. That region is discretized in internal cells and the corresponding integrals are transformed into integrals over the boundary of the cells. In addition, in this work, quadratic boundary elements and constant triangular internal cells are used.

It is also important to mention that, in this work, Cartesian tensor notation is used, with Greek indices varying from 1 to 2 and Latin ones from 1 to 3.

\section{BASIC FORMULATION}

Consider an isotropic, homogeneous and linearly elastic plate, with constant thickness $h$ and subjected to a transversal loadind $q$ per unit area, resting on a Pasternak-type foundation. The reaction of the foundation acting on the plate is represented by $p$ and expressed as:

$$
p=k u_{3}-k_{1}\left(u_{3,11}+u_{3,22}\right)
$$

in which:

$k=$ foundation modulus;

$k_{1}=$ shear foundation modulus;

$u_{3}=$ transverse displacement of a point of the plate;

$u_{3,11}$ and $u_{3,22}=$ second derivatives of the transverse displacement in a point of the plate, related to the axes $x_{1}$ and $x_{2}$, respectively.

Moments $M_{\alpha \beta}$ and shear forces $Q_{\alpha}$, including the contribution of elastic foundation reaction, are given by:

$$
\begin{gathered}
M_{\alpha \beta}=D \frac{(1-v)}{2}\left[u_{\alpha, \beta}+u_{\beta, \alpha}+\frac{2 v}{1-v} u_{\gamma, \gamma} \delta_{\alpha \beta}\right]+\frac{v(q-p)}{(1-v) \lambda^{2}} \delta_{\alpha \beta}, \\
Q_{\alpha}=D \frac{1-v}{2} \lambda^{2}\left(u_{\alpha}+u_{3},_{\alpha}\right),
\end{gathered}
$$

where:

$v=$ Poisson's ratio;

$\delta_{\alpha \beta}=$ Kronecker's delta;

$u_{\alpha}=$ rotations in the planes $x_{\alpha}-x_{3}$; 
$D=\frac{E h^{3}}{12\left(1-v^{2}\right)}=$ flexural rigidity of the plate;

$E=$ longitudinal elastic modulus;

$\lambda=\frac{\sqrt{10}}{h}=$ constant of Reissner's equations.

Equilibrium equations for a domain general point, obtained from the equilibrium of an infinitesimal plate element, are written as:

$$
\begin{gathered}
M_{\alpha \beta, \beta}-Q_{\alpha}=0, \\
Q_{\alpha, \alpha}+q-p=0 .
\end{gathered}
$$

For a boundary general point, the following boundary tractions are defined:

$$
\begin{aligned}
& p_{\alpha}=M_{\alpha \beta} n_{\beta}, \\
& p_{3}=Q_{\beta} n_{\beta},
\end{aligned}
$$

with $n_{\beta}$ being the direction cosines of the outward normal to the boundary.

In addition, the following boundary conditions are considered:

$$
\begin{aligned}
& u_{k}=\bar{u}_{k} \text { in } \Gamma_{u}, \\
& p_{k}=\bar{p}_{k} \text { in } \Gamma_{p},
\end{aligned}
$$

with $\Gamma_{u}$ being the part of the boundary where the generalized displacements are prescribed and $\Gamma_{p}$ being the part of the boundary where the generalized tractions are prescribed. The total boundary is defined as:

$$
\Gamma=\Gamma_{u} \cup \Gamma_{p} .
$$

\section{INTEGRAL EQUATIONS}

From a Weighted Residual Procedure and Reissner's plate bending theory, including the contribution of the elastic foundation reaction, and after performing the limits of the integrals as the source point tends to the boundary, the following integral equation can be obtained, which is valid for points situated in the domain $\Omega$ or at the boundary $\Gamma$ :

$$
c_{i j}(\xi) u_{j}(\xi)=\int_{\Gamma}\left[u_{i j}^{*}(\xi, x) p_{j}(x)-p_{i j}^{*}(\xi, x) u_{j}(x)\right] d \Gamma(x)+
$$




$$
\begin{aligned}
& +\int_{\Omega} q(x)\left[u_{i 3}^{*}(\xi, x)-\frac{v}{(1-v) \lambda^{2}} u_{i \alpha, \alpha}^{*}(\xi, x)\right] d \Omega(x)- \\
& -\int_{\Omega} p(x)\left[u_{i 3}^{*}(\xi, x)-\frac{v}{(1-v) \lambda^{2}} u_{i \alpha, \alpha}^{*}(\xi, x)\right] d \Omega(x),
\end{aligned}
$$

where $u_{i j}^{*}(\xi, x)$ and $p_{i j}^{*}(\xi, x)$ represent components of the displacement tensor of the fundamental solution for the problem and components of the corresponding traction tensor, respectively, in the direction $j$ of the field point $x$ corresponding to the action of a unit concentrated generalized force applied in the direction $i$ of the source point $\xi$.

The coefficient $c_{i j}(\xi)$ depends on boundary geometry at point $\xi$ and one has:

$c_{i j}=\delta_{i j}$ if $\xi$ is a domain point;

$c_{i j}=\delta_{i j} / 2$ if $\xi$ is a smooth boundary point.

The domain integrals can be transformed into boundary integrals for several kinds of loading. In the present work, the load $q(x)$ is considered as a uniformly distributed load, so that $q(x)=q=$ constant. In addition, the region over the elastic support is considered divided into constant triangular cells and it is admitted that $p(x)$ is uniformly distributed in each cell, so that $p(x)=p=$ constant.

In this case, using the divergence theorem in order to transforme domain integrals into boundary integrals, one obtains:

$$
\begin{aligned}
c_{i j}(\xi) u_{j}(\xi) & =\int_{\Gamma}\left[u_{i j}^{*}(\xi, x) p_{j}(x)-p_{i j}^{*}(\xi, x) u_{j}(x)\right] d \Gamma(x)+ \\
& +q \int_{\Gamma}\left[v_{i, \alpha}^{*}(\xi, x)-\frac{v}{(1-v) \lambda^{2}} u_{i \alpha}^{*}(\xi, x)\right] n_{\alpha}(x) d \Gamma(x)- \\
& -\sum_{c=1}^{N c} p \int_{\Gamma_{c}}\left[v_{i, \alpha}^{*}(\xi, x)-\frac{v}{(1-v) \lambda^{2}} u_{i \alpha}^{*}(\xi, x)\right] n_{\alpha}(x) d \Gamma(x) .
\end{aligned}
$$

Displacements at an internal point $\xi$ are obtained from Eq. (8), with $C_{i k}=\delta_{i k}$, so that:

$$
\begin{aligned}
u_{j}(\xi) & =\int_{\Gamma}\left[u_{i j}^{*}(\xi, x) p_{j}(x)-p_{i j}^{*}(\xi, x) u_{j}(x)\right] d \Gamma(x)+ \\
& +q \int_{\Gamma}\left[v_{i, \alpha}^{*}(\xi, x)-\frac{v}{(1-v) \lambda^{2}} u_{i \alpha}^{*}(\xi, x)\right] n_{\alpha}(x) d \Gamma(x)- \\
& -\sum_{c=1}^{N c} p \int_{\Gamma_{c}}\left[v_{i, \alpha}^{*}(\xi, x)-\frac{v}{(1-v) \lambda^{2}} u_{i \alpha}^{*}(\xi, x)\right] n_{\alpha}(x) d \Gamma(x) .
\end{aligned}
$$


For calculating moments and shear forces at an internal point $\xi$, the following boundary equations are obtained:

$$
\begin{aligned}
M_{\alpha \beta}(\xi) & =\int_{\Gamma} u_{\alpha \beta k}^{*}(\xi, x) p_{k}(x) d \Gamma(x)-\int_{\Gamma} p_{\alpha \beta k}^{*}(\xi, x) u_{k}(x) d \Gamma(x)+ \\
& +q \int_{\Gamma} w_{\alpha \beta}^{*}(\xi, x) d \Gamma(x)-\sum_{c=1}^{N c} p \int_{\Gamma_{c}} w_{\alpha \beta}^{*}(\xi, x) d \Gamma(x)+ \\
& +\frac{v}{(1-v) \lambda^{2}}(q-p) \delta_{\alpha \beta}, \\
Q_{\alpha}(\xi) & =\int_{\Gamma} u_{3 \beta k}^{*}(\xi, x) p_{k}(x) d \Gamma(x)-\int_{\Gamma} p_{3 \beta k}^{*}(\xi, x) u_{k}(x) d \Gamma(x)+ \\
& +q \int_{\Gamma} w_{3 \beta}^{*}(\xi, x) d \Gamma(x)-\sum_{c=1}^{N c} p \int_{\Gamma_{c}} w_{3 \beta}^{*}(\xi, x) d \Gamma(x)
\end{aligned}
$$

Expressions for the tensors corresponding to the fundamental solution are found in Van der Weeën [9] and Karam and Telles [3].

\section{NUMERICAL IMPLEMENTATION}

For the numerical solution of Eq. (8), the boundary $\Gamma$ is divided into elements, in which $u_{j}$ and $p_{j}$ are calculated by interpolating the nodal values. Then, this equation is written in discretized form for each nodal point $\xi$ at $\Gamma$, by substituting the integrals in $\Gamma$ by summations of integrals in $\Gamma_{j}$, with $\Gamma_{j}$ being the boundary of the element $j$. Then, a system of $\mathrm{N}$ algebric equations is obtained that contains $\mathrm{N}$ displacement nodal values and $\mathrm{N}$ traction nodal values.

So, Eq. (8) can be written in the following discretized form:

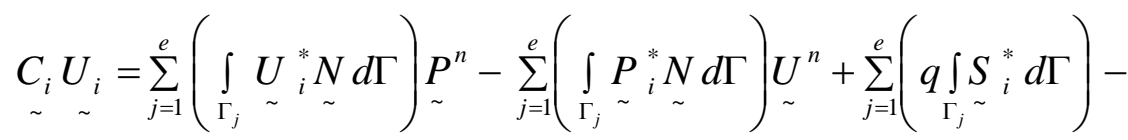

$$
\begin{aligned}
& -\sum_{c=1}^{N_{c}} \sum_{j=1}^{N_{e}} p\left(\int_{\Gamma_{c j}} \underset{\sim}{S_{i}^{*}} d \Gamma\right)
\end{aligned}
$$

where:

$\underset{\sim i}{C}=$ matrix which contains the coefficients $c_{i j}$ of Eq. (8);

$\underset{\sim i}{U}=$ vector with source point displacements;

$e=$ number of boundary elements; 
$N=$ matrix that contains the interpolation functions;

$\underset{\sim}{U_{i}^{*}}$ and $\underset{\sim}{P_{i}^{*}}=$ matrices that contain the components of tensors of the fundamental solution related to displacements and tractions, respectively;

$U^{n}$ and $P^{n}=$ vectors that contain the components of displacements and tractions, respectively, related to the nodal points of the considered element;

$N_{e}=$ number of boundary elements of each internal cell;

$N_{c}=$ total number of cells;

$\underset{\sim i}{S_{i}^{*}}=$ vector with components expressed as:

$$
S_{k}^{*}=\left(v_{k, \alpha}^{*}-\frac{v}{(1-v) \lambda^{2}} u_{k \alpha}\right) n_{\alpha}
$$

By applying Eq. (12) for all nodal points $\xi$ of the boundary, one obtains a system with a number of equations three times the number of nodal points, in the form:

$$
\underset{\sim}{H} \underset{\sim}{U}=\underset{\sim}{G} \underset{\sim}{P}+\underset{\sim}{B}-\underset{\sim}{M}
$$

where:

$U$ and $P=$ vectors that contain the nodal values of displacements and tractions, respectively;

$B=$ vector that contains the distributed load contribution;

$M=$ vector that contains the soil reaction contribution;

$H$ and $G=$ matrices that contain the integrals over the boundary elements.

The system of equations (14) admits as unknown displacements or tractions at boundary nodes. Since the reactions of the elastic support represent additional unknowns to this system, integral equations for transverse displacements of points situated in the geometric centre of internal cells and integral equations of the second derivatives of these transverse displacements are also added to this system.

By applying the integral equations of second derivatives of the transverse displacements relative to the axes $x_{1}$ and $x_{2}$ and the third of the equations represented in the expression presented in Eq. (8) to all cell points in which there exist contact with the elastic foundation, one has, after the discretization and combining with Eq. (14):

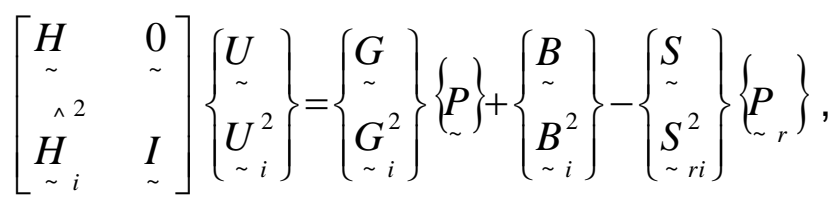

where index $i$ indicates that the vectors and matrices are related to internal points. 
By considering $p_{r}=k u_{3}-k_{1}\left(u_{3,11}+u_{3,22}\right)$ as the components of vector $\left\{\underset{\sim}{P_{r}}\right\}$, with $u_{3}, u_{3,11}$ and $u_{3,22}$ being unknowns, the system of equations can be written in the form:

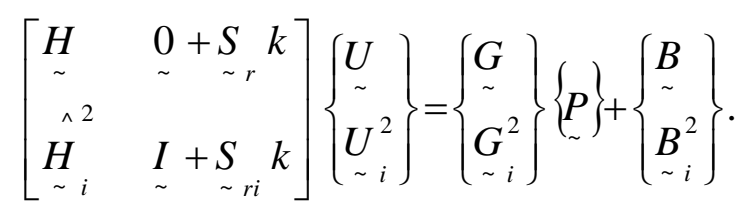

The values of displacements and tractions at boundary nodes are given, respectively, by vectors $U$ and $P$. For each nodal generalized direction, one must have the displacement or the traction with prescribed value and the other as unknown. In addition, the values of the transverse displacements and of the second derivatives of transverse displacements at internal cell points, contained in $\underset{\sim}{U_{i}^{2}}$, are also unknowns.

One can reorder the system represented by Eq. (16), so that all unknowns in $U, P$ and $\underset{\sim}{U_{i}^{2}}$ be allocated in a vector $\underset{\sim}{X}$ and all known values be allocated in a vector $F$. So, one obtains a system of the form:

$$
A X=F \text {. }
$$

By solving this system, the unknown values of displacements, tractions and second derivatives are obtained.

For calculating displacements at internal points, Eq. (9) is discretized and, for each point $\xi_{i}$ of region $\Omega$, one obtains:

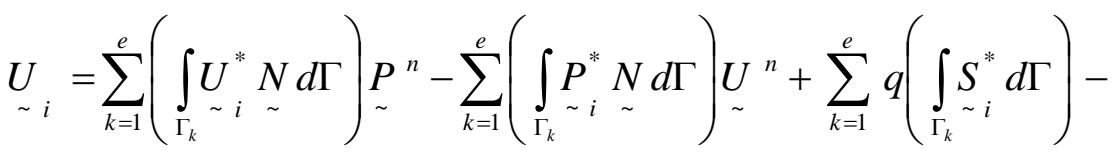

$$
\begin{aligned}
& -\sum_{c=1}^{N_{c}} \sum_{k=1}^{N_{e}} p\left(\int_{\Gamma_{c k}} \underset{\sim}{S^{*}} d \Gamma\right)
\end{aligned}
$$

For the calculation of moments and shear forces at internal points, Eqs. (10) and (11) are discretized and, for each internal point $\xi_{i}$, one has:

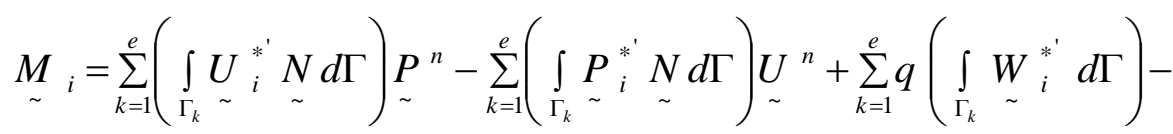

$$
\begin{aligned}
& -\sum_{c=1}^{N_{c}} \sum_{k=1}^{N_{e}} p\left(\int_{\Gamma_{c k}} \underset{\sim_{i}^{*}}{*_{i}^{\prime}} d \Gamma\right)+\frac{(q-p) \delta_{\alpha \beta}}{(1-v) \lambda^{2}}
\end{aligned}
$$




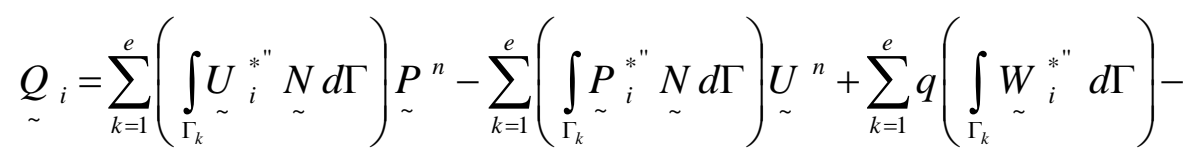

$$
\begin{aligned}
& -\sum_{c=1}^{N_{c}} \sum_{k=1}^{N_{e}} p\left(\int_{\Gamma_{c k}}^{W_{\sim}^{* *}} \underset{i}{W^{\prime \prime}} d \Gamma\right)
\end{aligned}
$$

\section{NUMERICAL EXAMPLES}

\subsection{Example 1: Clamped circular plate}

A circular plate with ratio $a$, clamped at the boundary and layed on elastic support in the domain is analysed in this example. Two discretizations are considered: the first one with 128 constant triangular cells and the second one with 200 constant triangular cells, according to Fig. 1. This plate is supposed to have $h / a=0.005$ and $v=0.3$. It is also considered that $k=\bar{k} D / a^{4}$, with $\bar{k}=200$; and $k_{1}=\overline{k_{1}} D / a^{2}$, with $\bar{k}_{1}=5$ and $\bar{k}_{1}=20$.

In order to prove the validity of the formulation, the results obtained in the present example are compared to numerical results of Jianguo et al. [2] and also with the analytical solution for thin plates obtained by Yu [10] and presented in Jianguo et al. [2], as they can be seen in Table 1, which presents the values obtained for the transverse displacements $u_{3}=10^{2} \overline{u_{3}} D / q a^{4}$ and for the moments $M_{r r}=10^{2} \overline{M_{r r}} / q a^{2}=M_{t t}=10^{2} \overline{M_{t t}} / q a^{2}$ at the plate centre. It can be verified that the results are in accordance.

In Fig. 2, one can observe the variation of the transverse displacements along a ratio of the plate and in Figs. 3 and 4 , considering $\bar{k}_{1}=5$ and $\bar{k}_{1}=20$, respectively, one can observe the variation of moments $\mathrm{M}_{\mathrm{rr}}$, also in radial direction.
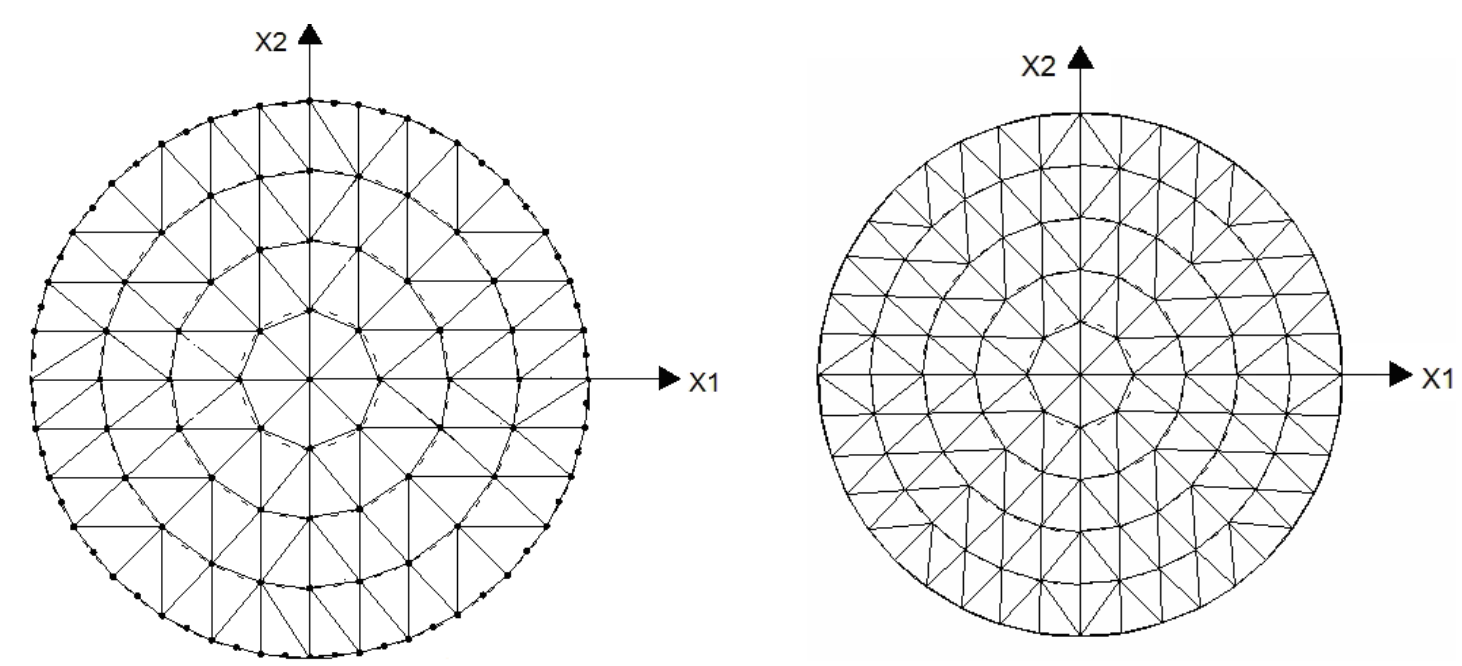

Figure 1. Boundary and domain discretization of a circular plate 
Table 1. Results for displacements and moments at the centre of the circular plate

\begin{tabular}{|c|c|c|c|c|}
\cline { 2 - 5 } \multicolumn{1}{c|}{} & \multicolumn{2}{|c|}{$\bar{k}_{1}=5$} & \multicolumn{2}{|c|}{$\bar{k}_{1}=20$} \\
\cline { 2 - 5 } & $\frac{\overline{u_{3}} D 10^{2}}{q a^{4}}$ & $\frac{\overline{M_{r r}} 10^{2}}{q a^{2}}=\frac{\overline{M_{t t}} 10^{2}}{q a^{2}}$ & $\frac{\overline{u_{3}} D 10^{2}}{q a^{4}}$ & $\frac{\overline{M_{r r}} 10^{2}}{q a^{2}}=\frac{\overline{M_{t t}} 10^{2}}{q a^{2}}$ \\
\hline $\begin{array}{c}\text { Present work } \\
128 \text { cells }\end{array}$ & 0.4490297 & 1.720840 & 0.3426192 & 1.185250 \\
\hline $\begin{array}{c}\text { Present work } \\
\text { 200 cells }\end{array}$ & 0.4474958 & 1.707700 & 0.3410063 & 1.180700 \\
\hline Jianguo et.al. [2] & 0.4447239 & 1.676206 & 0.3383348 & 1.162126 \\
\hline $\begin{array}{c}\text { Analytical } \\
\text { solution for thin } \\
\text { plates [10] }\end{array}$ & 0.4448609 & 1.675682 & 0.3384423 & 1.161785 \\
\hline
\end{tabular}

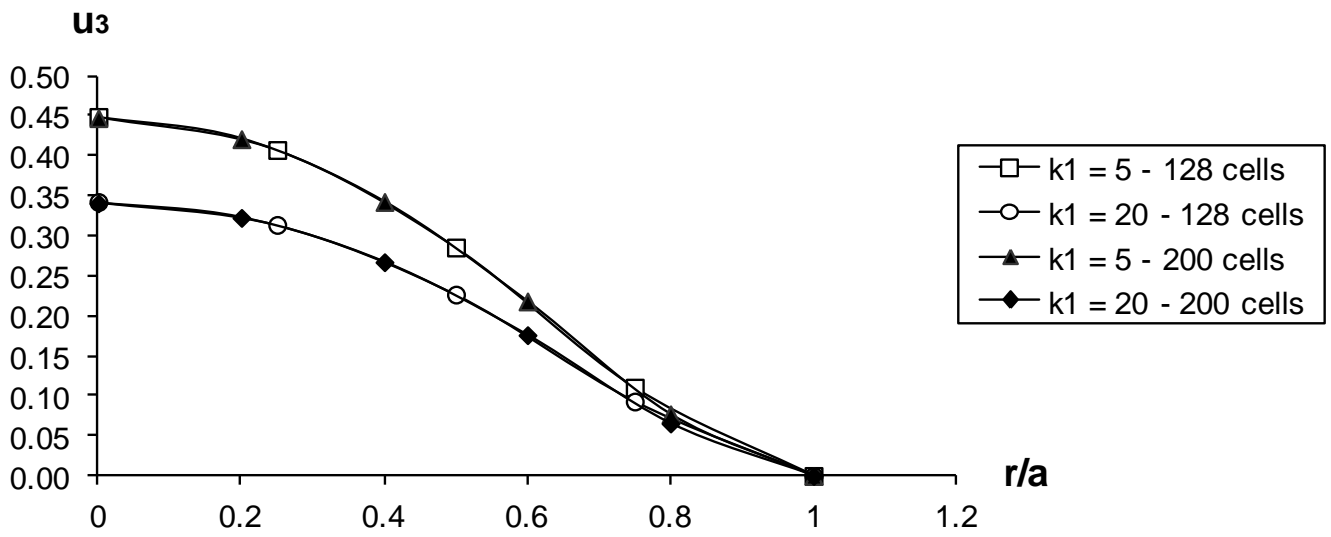

Figure 2. Variation of transverse displacement $u_{3}$ along $x_{1}$

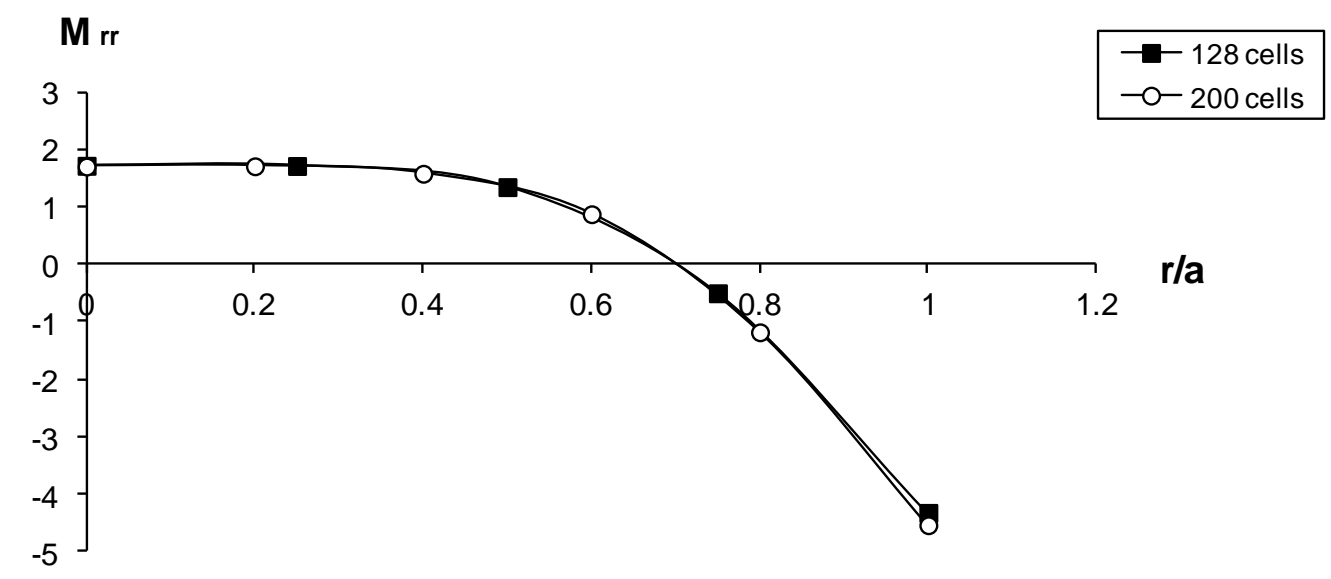

Figure 3. Variation of moment $M_{r r}$ along $x_{1}$, considering $\bar{k}_{1}=5$ 


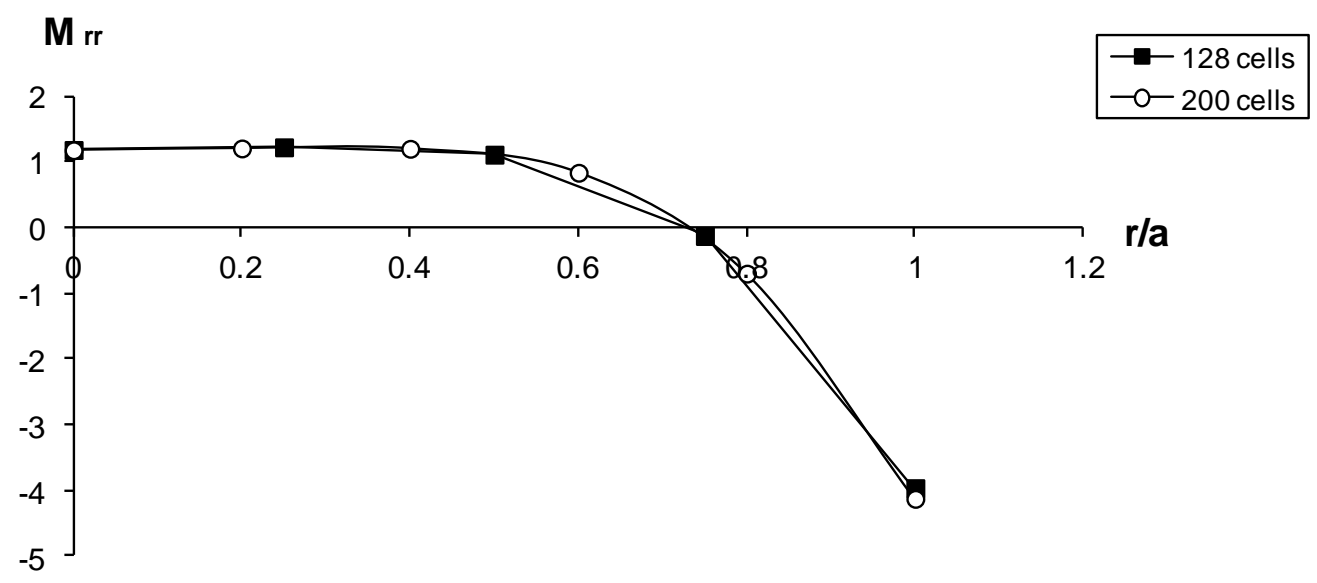

Figure 4. Variation of moment $M_{r r}$ along $x_{1}$, considering $\bar{k}_{1}=20$

\subsection{Example 2: Simply supported rectangular plate}

In this example, a rectangular plate simply supported at the boundary is considered, having total or parcial elastic support in his domain. The foundation modulus, $k$, and the shear foundation modulus, $k_{1}$, vary in the plate domain (Fig. 5). The boundary is discretized into 12 quadratic elements and the domain into 32 constant triangular cells (Fig. 6). It is admitted that $h / a=0.05$ and $v=0.3$. In addition, it is considered that $k=\left(\bar{k} D / a^{4}\right)$, with $\bar{k}$ $=100$ and $\bar{k}=200$; and $k_{1}=\left(\overline{k_{1}} \mathrm{D} / \mathrm{a}^{2}\right)$, with $\bar{k}_{1}=20$ and $\bar{k}_{1}=50$.

In Fig. 7, one can observe the variation of the transverse displacement along $x_{2}=a / 2$ and, in Fig. 8, the variation of flexural moments $\mathrm{M}_{11}$ and $\mathrm{M}_{22}$ along the same line.

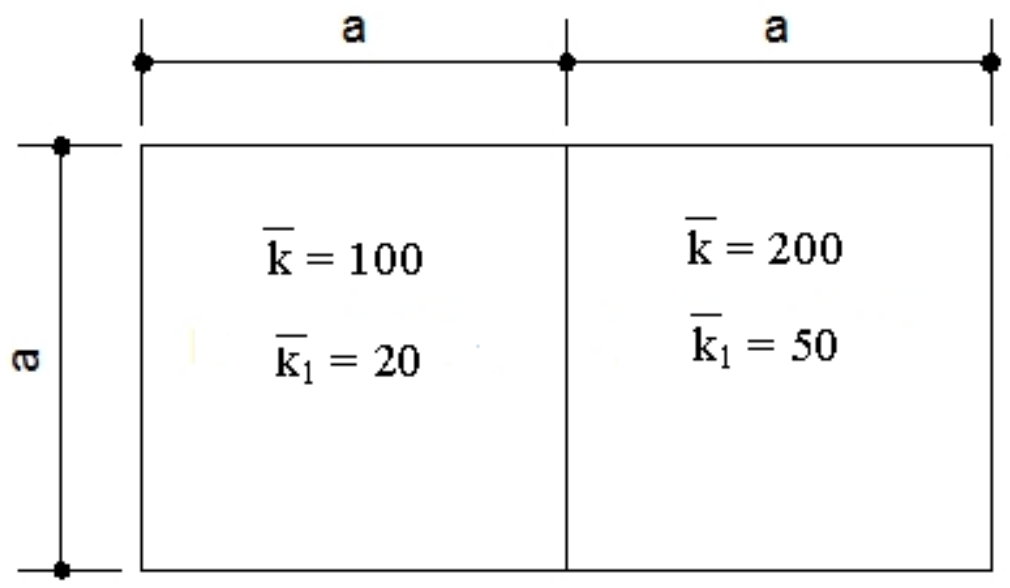

Figure 5. Rectangular plate simply supported at the boundary 


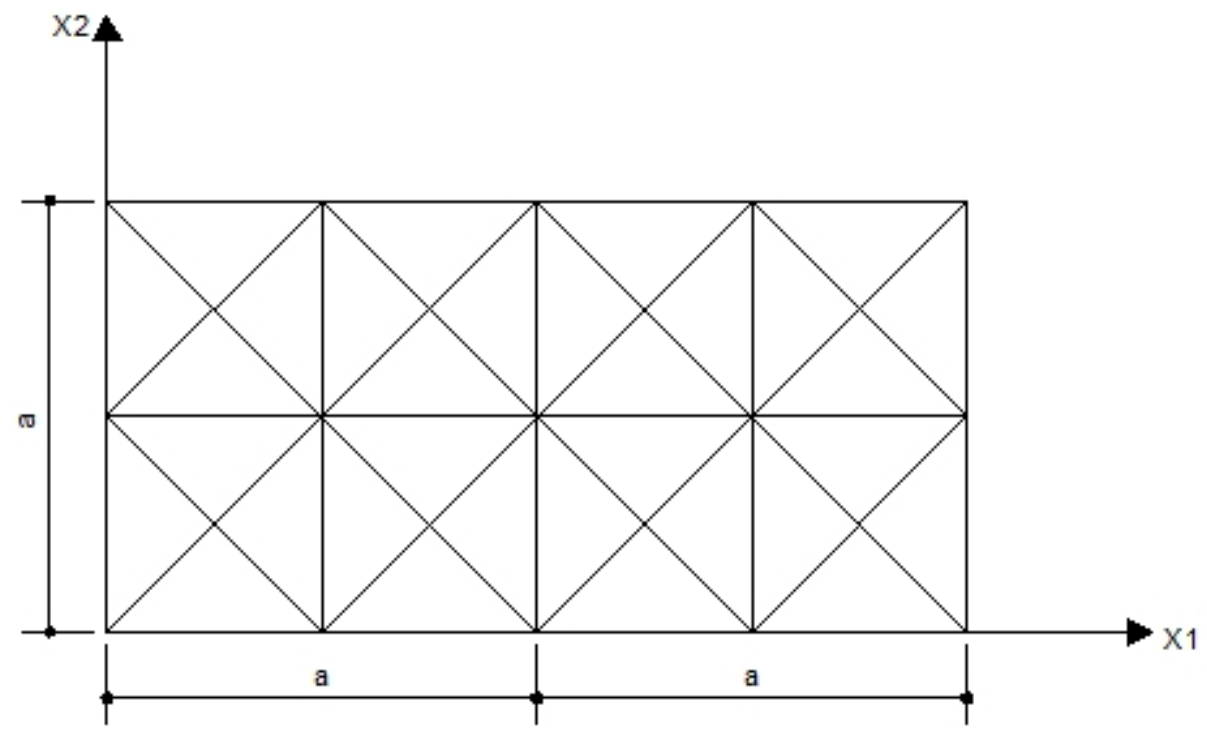

Figure 6. Boundary and domain discretization of a rectangular plate

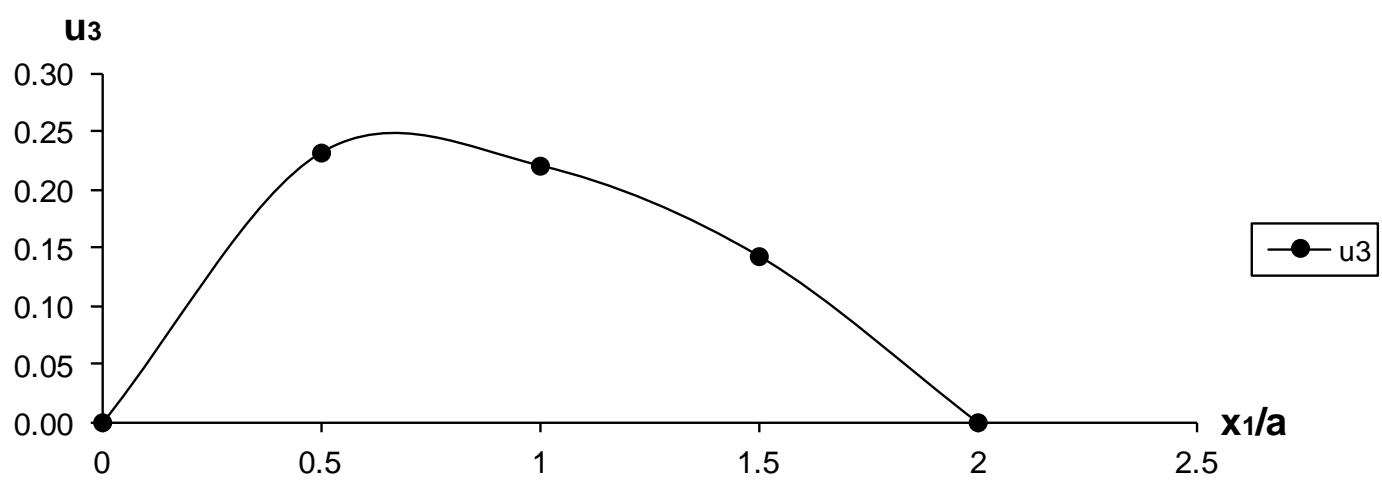

Figure 7. Variation of transverse displacement $u_{3}$ along $x_{2}=a / 2$

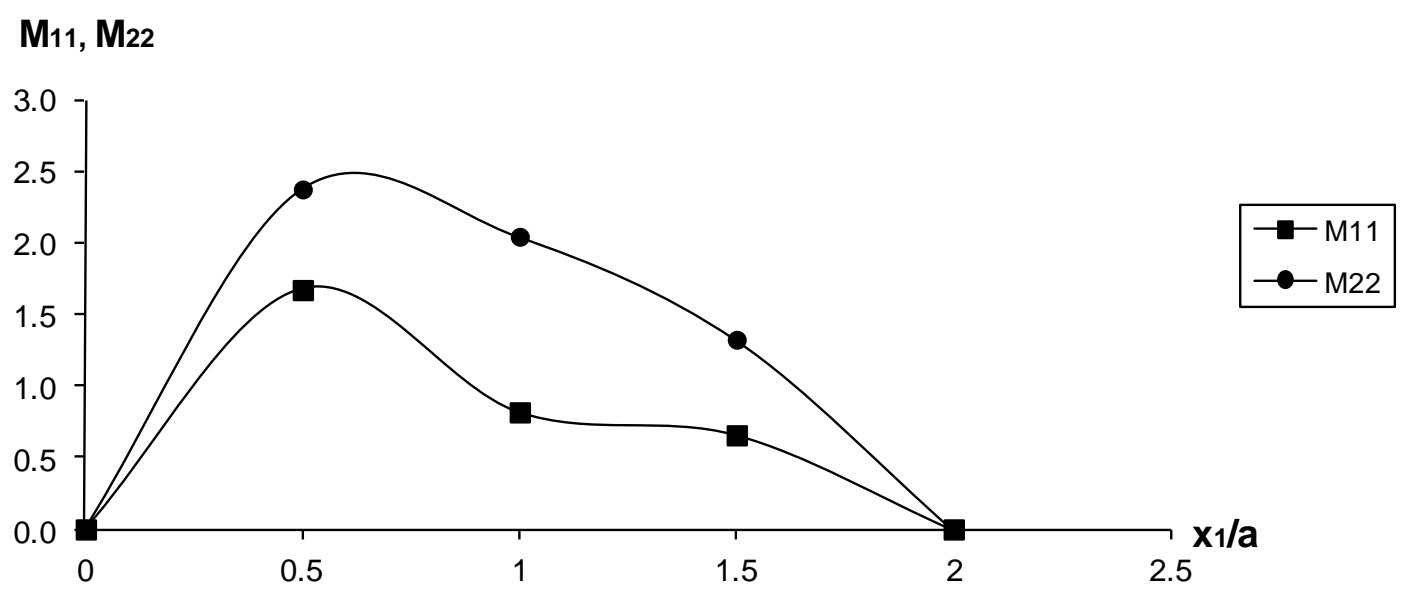

Figure 8. Variation of moments $M_{11}$ and $M_{22}$ along $x_{2}=a / 2$ 


\section{CONCLUSIONS}

A formulation for analysis of plates resting on elastic foundation using the BEM was presented in this work, with Pasternak's foundation model and considering Reissner's theory for plate bending. A procedure that considers the transverse displacements and their second derivatives as unknowns at cell points was used. The obtained results were compared with those obtained from the literature. It was observed that they are in accordance and this allows for one to verify the validity of the presented formulation. It is also important to observe that this approach allows the analysis of plates with modulus of foundation varying in the domain.

\section{Acknowledgements}

The authors acknowledge the financial support from UENF/FAPERJ for this work.

\section{REFERENCES}

[1] Altoé, M., Karam, V. J., “Análise de placas apoiadas sobre base elástica utilizando o Método dos Elementos de Contorno com o modelo de fundação de Pasternak". XXX Cilamce: Iberian Latin American Congress on Computational Methods in Engineering, 2009.

[2] Jianguo, W., Xiuxi, W., Maokuang, H., "Fundamental Solutions and Boundary Integral Equations for Reissner's Plates on Two Parameter Foundations". International Journal of Solids and Structures, 29, 1233-1239, 1992.

[3] Karam, V. J., Telles, J. C. F., “On Boundary Elements for Reissner's Plate Theory”. Engineering Analysis, 5(1), 21-27, 1988.

[4] Paiva, J. B., “Aplicação do Método dos Elementos de Contorno à análise de placas apoiadas em base elástica". X Congresso Ibero-Latino-Americano sobre Métodos Computacionais em Engenharia, Portugal, A47 - A59, 1989.

[5] Rashed, Y. F., Aliabadi, M. H., Brebbia, C. A., "The Boundary Element Method for Thick Plates on a Winkler Foundation". International Journal for Numerical Methods in Engineering, 41, 1435-1462, 1998.

[6] Rashed, Y. F., Aliabadi, M. H., Brebbia, C. A., "A Boundary Element Formulation for a Reissner Plate on a Pasternak Foundation”. Computers and Structures, 70, 515-532, 1999.

[7] Reissner, E., "The Effect of Transverse Shear Deformation on the Bending of Elastic Plates". Journal of Applied Mechanics. 12, A69-A77, 1945. 
[8] Ribeiro, N. S., Karam, V. J., “Análise de placas apoiadas sobre base elástica pelo Método dos Elementos de Contorno com o modelo de fundação de Winkler". XXX Cilamce: Iberian Latin American Congress on Computational Methods in Engineering, 2009.

[9] Van der Weeën, F., “Application of the Direct Boundary Element Method to Reissner's Plate Model”. Boundary Elements in Engineering, 487-499, 1982.

[10] Yu, Y. Y., "Axisymmetric bending of circular plates under simultaneous action of lateral load, Force in the Middle Plane and Elastic Foundation". Journal of Applied Mechanics, 24,141-143, 1957. 